\title{
Enfermedad tromboembólica venosa: análisis de 239 casos
}

\author{
M. C. MUÑOZ LÓPEZ DE RODAS, M. SERRANO CAZORLA, L. DÍAZ VIDAL, \\ R. CIGÜENZA GABRIEL, J. ANTOLÍN ARIAS
}

Servicio de Medicina Interna I. Hospital Clínico San Carlos. Madrid

THROMBOEMBOLIC DISEASE: ANALYSIS OF 239 CASES.

\begin{abstract}
RESUMEN
Objetivo: Conocer el número de pacientes que ingresan o que desarrollan durante su ingreso en un hospital ETEV, analizar el manejo de esta enfermedad y sentar las bases para un estudio prospectivo de dicha enfermedad.

Métodos: Se trata de un estudio descriptivo retrospectivo de pacientes diagnosticados de ETEV durante su ingreso en el Hospital Clínico San Carlos de Madrid en un período de 6 meses. Se recogen los datos relacionados con antecedentes epidemiológicos, diagnóstico, tratamiento y complicaciones de los pacientes con trombosis venosa profunda (TVP), tromboembolismo pulmonar (TEP) o ambas (TVP + TEP).

Resultados: Del 1 de octubre de 2003 al 31 de marzo de 2004, 239 pacientes fueron diagnosticados de ETEV (64 TVP, 125 TEP y 51 TVP + TEP) al alta en nuestro hospital con una edad media de 73,2 años (desviación estándar 13,64). Como factores de riesgo con diferencias estadísticamente significativas detectamos la quimioterapia, el infarto agudo de miocardio y la obesidad. No se ha observado relación entre el tipo de tratamiento utilizado y la aparición de hemorragia. Los pacientes con episodios previos de ETEV presentaron hemorragias más frecuentemente que aquellos sin dichos antecedentes. La hipocinesia de ventrículo derecho en el ecocardiograma fue un factor de mal pronóstico para la muerte por ETEV así como la aparición de TVP + TEP.

Conclusión: En más del 50\% de los pacientes la ETEV era TEP y más del $60 \%$ eran mujeres. Es importante disponer de información sobre estos pacientes, ya que $2 / 3$ ingresan en medicina interna. La quimioterapia, el IAM y la obesidad fueron factores que se asociaron a TVP, TEP y TVP + TEP de forma significativa, respectivamente. La hipocinesia de ventrículo derecho en el ecocardiograma fue un factor de mal pronóstico para la muerte por ETEV así como la coincidencia de TVP + TEP.
\end{abstract}

PALABRAS CLAVE: Embolismo pulmonar. Tromboembolismo pulmonar. Trombosis venosa profunda.

\section{ABSTRACT}

Aim: To know the number of patients that are admitted in the hospital with TED or those who have developed it during their stay, analyzing how to manage this disease and make a basis for a prospective study of this disease.

Methods: It is a descriptive and retrospective study of TED diagnosed patients during their stay at the Hospital Clínico San Carlos of Madrid for a 6 month period. Data related with epidemiologic records, diagnosis, treatment and complications of patients with Deep-Vein Thrombosis (DVT), Pulmonary Thromboembolism (PTE) or both (DVT+PTE) are collected.

Results: From October 1st of 2003 to March 31st of 2004, 239 patients were diagnosed with TED (64 DVT, 125 PTE y 51 DVT + PTE) when they were discharged from our hospital, with an average age of 73.2 years (standar desviation 13.64). We classify as risky factors with significative statistical diferences chemotherapy, acute myocardium infarction and obesity. It has not been found any relation between the treatment used and the development of hemorrage. Patients with previous episodies of TED had more frequent hemorragies than those without such records. Hypokinesia in the right ventricle shown on the echocardiogram supposed a gloomy prognosis of the death for TED as well as the development of DVT + PTE.

Conlusion: In more than a $50 \%$ of patients, TED was PTE and more than a $60 \%$ were women. It is important to obtain information about these patients because $2 / 3$ of them are admitted to internal medicine. Chemotherapy, AIM and obesity were factors significatively associated to DVT, PTE and DVT + PTE. Hypokinesia in the right ventricle shown on the echocardiogram supposed a gloomy prognosis for TED as well as the development of DVT + PTE.

KEY WORDS: Pulmonary Embolism. Pulmonary Thromboembolism. Deep-Vein Thrombosis.

Muñoz López de Rodas MC, Serrano Cazorla M, Díaz Vidal L, Cigüenza Gabriel R, Antolín Arias J. Enfermedad tromboembólica venosa: análisis de 239 Casos. An Med Interna (Madrid) 2006; 23: 565-568.

\section{INTRODUCCIÓN}

La enfermedad tromboembólica venosa (ETEV) comprende dos entidades: la trombosis venosa profunda (TVP) y el tromboembolismo pulmonar (TEP). En España hay pocos estudios sobre esta entidad por lo que no es posible saber a ciencia cierta su incidencia real. En otros países occidentales se estima en 1 por 1.000 habitantes y año, y aumenta con la edad hasta llegar a un caso por cada 100 personas y año a los 85 años $(1,2)$.

Trabajo aceptado: 5 de julio de 2006 
Es una enfermedad frecuente en el ámbito hospitalario, de difícil diagnóstico en ocasiones puesto que puede presentarse de diversas formas y potencialmente mortal (hasta el 12\% de los pacientes fallecen en los primeros 30 días) (1), por lo que es necesario identificarla y tratarla correctamente a tiempo. Además, el diagnóstico y tratamiento tempranos mejoran la supervivencia y disminuyen la recurrencia (3). La anticoagulación, que es la base del tratamiento, tampoco está exenta de riesgos ya que, si es insuficiente se corre el riesgo de la recidiva y si es excesivo, el de la hemorragia.

\section{MATERIAL Y MÉTODOS}

Se incluyen los casos nuevos que son diagnosticados de ETEV en el hospital en un período de 6 meses, es decir, que son ingresados por dicha enfermedad o que aparece como complicación durante su ingreso por otro motivo. Se recogen los datos relacionados con antecedentes epidemiológicos, diagnóstico, tratamiento y complicaciones de los pacientes con trombosis venosa profunda, tromboembolismo pulmonar o ambas.

Casos de ETEV: trombosis venosa profunda, tromboembolismo pulmonar o ambas (TVP + TEP) diagnosticados en el Hospital Clínico San Carlos de Madrid del 1 de Octubre de 2003 al 31 de Marzo de 2004. Son diagnosticados por la positividad para alguna prueba diagnóstica (Eco-Doppler de miembros, flebografía, gammagrafía pulmonar de ventilación-perfusión o TC helicoidal) o por alta sospecha clínica sin posibilidad de confirmación.

\section{ANÁLISIS ESTADÍSTICO}

Las variables cualitativas se presentan con su distribución de frecuencias. Las variables cuantitativas se resumen con su media, desviación estándar (DE).

Se evaluó la asociación entre variables cualitativas con el test de Chi-cuadrado o prueba exacta de Fisher, en el caso de que más de un $25 \%$ de los esperados fueran menores de 5 . Para todas las pruebas se aceptó un valor de significación del 5\%. El procesamiento y análisis de los datos se realizó mediante el paquete estadístico SPSS 12.0.

\section{RESULTADOS}

Del 1 de octubre de 2003 al 31 de marzo de 2004, 239 pacientes (148 mujeres y 91 hombres) fueron diagnosticados de ETEV al alta en el Hospital Clínico San Carlos de Madrid (66,1\% en el servicio de Medicina Interna), con una edad media de 73,2 años (desviación estándar 13,64). Las características clínico-epidemiológicas se muestran en la Tabla I. En 64 pacientes $(26,9 \%)$, la clínica de presentación fue de TVP, siendo la localización más frecuente en miembros inferiores $(79,1 \%)$, y en dos casos, de forma bilateral $(0,8 \%)$. En 124 pacientes $(51,7 \%)$ se manifestó como TEP y los 51 restantes $(21,4 \%)$ lo hizo como TVP + TEP. Como factores de riesgo con diferencias estadísticamente significativas encontramos la quimioterapia $(44,7 \%$ de las TVP la recibían, $\mathrm{p}<0,01)$, el infarto agudo de miocardio (IAM) $(13,7 \%$ de los TEP lo habían tenido, $\mathrm{p}<0,01)$ y la obesidad $(14,9 \%$ de los pacientes con TVP + TEP eran obesos, $\mathrm{p}<0,05$ ).
TABLA I

CARACTERÍSTICAS CLÍNICO-EPIDEMIOLÓGICAS DE LA SERIE

\begin{tabular}{lr}
\hline & $n=239$ \\
\hline EPOC & $30(13,4 \%)$ \\
IC & $19(8,5 \%)$ \\
IAM & $18(8 \%)$ \\
ACV & $15(6,7 \%)$ \\
obesidad & $19(8,5 \%)$ \\
Prótesis & $13(5,8 \%)$ \\
Quimioterapia & $28(11,7 \%)$ \\
Inmovilización & $66(27,8 \%)$
\end{tabular}

EPOC: enfermedad pulmonar obstructiva crónica, IC: insuficiencia cardiaca, IAM: infarto agudo de miocardio, ACV: accidente cerebrovascular.

El diagnóstico de TEP se realizó mediante Gammagrafía pulmonar en el 70,2\% y por TC helicoidal en el 18,5\%. En las TVP el diagnóstico se hizo por Eco-Doppler en el 73,4\%. Los D-Dímeros se realizaron en el $40 \%$ de los pacientes, siendo negativos en el 10,4\% (en el 12,9\% de los pacientes con TEP y $4 \%$ de los pacientes con TVP + TEP). Sólo en el $21 \%$ se solicitaron Anticuerpos anticardiolipina (ACA) (más frecuentemente en pacientes que presentan TVP + TEP que en el resto de los casos, con diferencias estadísticamente significativas, $\mathrm{p}<0,01)$ y en ningún caso antitrombina-III, proteína $\mathrm{C}$ ni proteína $S$.

En cuanto al tratamiento, el 42,1\% recibió heparina sódica en perfusión como tratamiento anticoagulante en la fase aguda frente al $58,1 \%$, en los que se utilizó heparina de bajo peso molecular (HBPM). En el 2,9\% se puso filtro de cava y en ningún paciente se realizó fibrinolisis ni embolectomía. En la fase crónica, el $60,4 \%$ se anticoaguló con anticoagulantes orales y el $36,4 \%$ con HBPM.

Presentaron complicaciones hemorrágicas 20 pacientes $(8,8 \%)$, siendo el sangrado digestivo la localización más frecuente $(44,4 \%)$. No se han encontrado diferencias estadísticamente significativas en cuanto al tipo de tratamiento utilizado y la aparición de hemorragia. En nuestro estudio, el 16,3\% de los pacientes que tenían antecedentes de ETEV presentaron complicaciones hemorrágicas con diferencias estadísticamente significativas $(\mathrm{p}<0,05)$.

El 19,2\% de los pacientes falleció durante el ingreso habiéndose encontrado diferencias estadísticamente significativas en relación con la clínica de presentación (27,5\% de las TVP + TEP, $p<0,05)$ y con la hipocinesia del ventrículo derecho $(27,3 \%$ de los pacientes con dicha alteración fallecen a lo largo del ingreso, $\mathrm{p}<0,05)$.

\section{DISCUSIÓN}

La ETEV afecta preferentemente a ancianos, que por otra parte, son los que mayor prevalencia de enfermedad cardiopulmonar crónica presentan, lo que dificulta el diagnóstico precoz (4). Los diagnósticos equívocos que con más frecuencia solapan al TEP son la insuficiencia cardiaca y la neumonía (3). En nuestro estudio, la edad media de los pacientes fue de 73,2 años, siendo el sexo femenino el más afectado, al contrario que en otras series (2) pero sin llegar a encontrar diferencias estadísticamente significativas. 
En el estudio RIETE (5), fue mayor el porcentaje de TVP $(69,6 \%)$ que de TEP $(18,8 \%)$. Sin embargo, en nuestro hospital la forma clínica más frecuente fue el TEP $(51,7 \%)$, seguida de la TVP (26,9\%). Esto es debido a que en nuestro medio la mayor parte de TVP se tratan en Hospitalización Domiciliaria sin ingreso.

La patogenia se basa en la tríada de Virchow: hipercoagulabilidad, estasis venoso y lesión endotelial. Los factores de riesgo asociados pueden ser congénitos (déficit de Antitrombina-III, déficit de Proteína $\mathrm{C}$ y de Proteína $\mathrm{S}$, mutación del factor $\mathrm{V}$ de Leiden...) o adquiridos (inmovilidad, obesidad, cirugía traumatológica en los dos meses previos, viajes de larga duración, neoplasias...) (1). En nuestros pacientes observamos la relación, estadísticamente significativa, entre el tratamiento quimioterápico, el IAM y la obesidad y la aparición de TVP, TEP y TVP + TEP respectivamente.

En cuanto al diagnóstico de TVP, la técnica más utilizada fue el Eco- doppler de miembros inferiores, que actualmente se considera la técnica de elección. La flebografía sigue siendo el "gold standard" aunque se reserva para los casos dudosos, necesidad de confirmación diagnóstica, evaluación de la trombolisis o inserción de filtros de cava ya que es una prueba que conlleva ciertos riesgos (6). En nuestro estudio se utilizó en el 4,6 \% de los pacientes. Para el diagnóstico de TEP la técnica más utilizada fue la Gammagrafía pulmonar de ventilación-perfusión $(70,2 \%)$ seguida de la TC helicoidal. Los D-dímeros se realizaron en el $40 \%$ de los pacientes, siendo negativos en el 10,4\% de ellos pese a que al alta fueron diagnosticados de ETEV. Esto demuestra que si la sospecha clínica es elevada hay que agotar todas las posibilidades diagnósticas antes de descartar una enfermedad tan grave. En ningún caso se solicitó durante el ingreso el estudio de coagulación para descartar trombofilia congénita, si bien se informaba al paciente de la realización de dicho análisis de forma ambulatoria una vez pasada la fase aguda de la enfermedad. Este hecho puede ser debido a que hay que iniciar el tratamiento en cuanto se tenga alta sospecha de ETEV, que en numerosas ocasiones aparece en los servicios de urgencias o en horario de tarde-noche cuando no es posible solicitarlo. Además, hay que tener en cuenta que en los pacientes heparinizados carecen de valor las determinaciones de antitrombina, y en los tratados con anticoagulantes orales, los de proteína C y S (6).

En cuanto al tratamiento, puede haber diferentes estrategias: anticoagulación, filtro de vena cava, trombolisis o trombectomía quirúrgica. Diferenciamos dos fases: aguda (sospecha clínica de ETEV o primeros días del diagnóstico) y crónica (previo al alta o tras varias semanas de diagnóstico). $\mathrm{La}$ anticoagulación fue el tratamiento más utilizado en ambas fases, ya fuese con heparina sódica o con HBPM. En el 2,9\% de los pacientes se puso un filtro de vena cava y en ninguno se realizó fibrinolisis ni trombectomía. Al alta hubo un claro predominio de los anticoagulantes orales (ACO) frente a las HBPM (el 60,4\% frente al 36,4\%). Hay estudios que señalan la posibilidad de realizar el tratamiento de la TVP de miembros inferiores (de localización proximal o distal) con HBPM a cargo de unidades de Hospitalización a Domicilio sin necesidad de que el paciente permanezca ingresado, de forma segura y eficaz (con porcentajes de sangrado o reingreso similar a si el tratamiento se hubiese completado en el hospital) con la ventaja que ello supone para el paciente, ya que el número de controles de coagulación que precisan suele ser menor y está más cómodo en su casa (7).

Pueden aparecer dos tipos de complicaciones durante el tratamiento anticoagulante: la recidiva de la enfermedad (por anticoagulación insuficiente) o hemorragia (por exceso de dosis). No hemos encontrado diferencias estadísticamente significativas entre el tratamiento anticoagulante utilizado y la aparición de hemorragia. La aparición de dicha complicación en nuestros pacientes $(8,8 \%)$ es bastante similar a la obtenida en otros estudios (entre 5,3\% y 12,5\%) (5,8). Los pacientes con episodios previos de ETEV presentaron hemorragias más frecuentemente que aquellos sin dichos antecedentes, posiblemente esto puede ser debido a que se anticoagule con mayores dosis a este tipo de pacientes pensando que la causa de la recidiva sea la anticoagulación insuficiente.

La mortalidad intrahospitalaria atribuida a la ETEV sigue siendo elevada (9). En el estudio RIETE fallecieron el 4,7\% de los pacientes que se siguieron a lo largo de tres meses, encontrando una relación estadísticamente significativa con la aparición de hipoprotrombinemia (5). En otros estudios se estima que la mayoría de los fallecimientos ocurren durante el primer año de seguimiento y que se mantiene estable durante los años siguientes con una frecuencia del $10 \%$ aproximadamente (10). En nuestro estudio encontramos una mortalidad muy superior (el 19,2\% de los pacientes fallecieron) habiéndose encontrado diferencias estadísticamente significativas en relación con la clínica de presentación (TVP + TEP) y con la hipocinesia del ventrículo derecho. No obstante, la población estudiada es muy diferente, con mucha mayor proporción de TEP sobre TVP en nuestro caso (ya que estas últimas se tratan en Hospitalización Domiciliaria sin ingreso). El ecocardiograma nos permite hacer el diagnóstico diferencial entre el TEP y el taponamiento pericárdico, la disección de aorta y el IAM y además, nos permite valorar el pronóstico del TEP submasivo ya que los signos de disfunción del ventrículo derecho (hipocinesia moderada o grave), la hipertensión pulmonar, la presencia de trombos móviles en cavidades derechas o la persistencia del foramen oval abierto se han identificado como marcadores de gravedad $(1,6,11)$.

\section{Bibliografía}

1. Uresandi F, Blanquer J, Conget F, de Gregorio MA, Lobo JL, Otero R, et al. Guía para el diagnóstico, tratamiento y seguimiento de la tromboembolia pulmonar. Arch Bronconeumol 2004; 40: 580-94.

2. White RH. The epidemiology of venous thromboembolism. Circulation 2003; 107: 14-8.

3. Corral Gudino L, Guijo Hernández T, Moreiro Barroso M, Cordero M,
Pérez Arellano JL y Del Pino Montes J. Tromboembolia pulmonar: análisis de los casos sin sospecha inicial y sensibilidad de tres modelos clínicos. Med Clin (Barc) 2003; 121: 601-5.

4. Calvo Romero JM, Pérez Miranda M, Bureo Dacal P. Tromboembolismo pulmonar en el anciano. An Med Interna (Madrid) 2003; 20: 1; $21-4$. 
5. Suárez Fernández C, González-Fajardo JA, Monreal Bosch M y grupo del registro (RIETE). Registro informatizado de pacientes con enfermedad tromboembólica en España (RIETE): justificación, objetivos, métodos y resultados preliminares. Rev Clin Esp 2003; 203: 68-73.

6. Gabriel Botella F. Reflexiones sobre la enfermedad tromboembólica venosa. An Med Interna (Madrid) 2003; 20: 9; 447-50.

7. Álvarez-Nebreda, M L, Pérez-Tamayo I, Brañas-Baztán F, Rada-Martínez S, Serra-Rexach J A, González-Ramallo, et al. Tratamiento de la trombosis venosa profunda en pacientes mayores de 60 años en una unidad de hospitalización a domicilio. Rev Esp Geriatr Gerontol 2002; 37: 254-9.

8. Calvo Romero JM, Arévalo lorido JC, Romero Requena J, Pérez Alonso JL, Ortiz Descane C, Gutiérrez Montaño C, et al. Enfermedad trom- boembólica venosa en un área rural del sur de Extremadura (España). An Med Interna (Madrid) 2005; 22: 10; 465-8.

9. Calvo Romero JM, Arrebola García JD, Magro Ledesma D, Ramos Salado JL, Bureo Dacal JC, Bureo Dacal P, et al. El espectro clínico actual del tromboembolismo pulmonar. Rev Clin Esp 2002; 202: 130-5.

10. Echegaray Agara M, Alonso Martínez JL, Urbieta Echezarreta MA, Abínzano Guillén ML, González Arencibia C, Solano Remírez M. Tromboembolismo pulmonar: evolución a largo plazo y epidemiología clínica. An Med Interna (Madrid) 2003; 20: 9; 451-6.

11. Goldhaber SZ. Pulmonary Embolism. N Engl J Med 1998; 339: $93-$ 104. 